$\pm \mathbb{E}$

Global Journals Inc.

$\rightarrow \infty$

$\rightarrow$

Online ISSN: 2249-4626 \& Print ISSN: 0975-5896

\title{
Time Dilation and Special Relativity
}

By Svanik Garg

Abstract- The presentation and defination of Time as a concept in the Theory Special Theory of Relativity is remarkably difference to that of Time in the Theory of Classical Mechanics. In Special Relativity, Time is considered as the fourth dimension in the fabric of spacetime. However, the presentation of time as such, can be argued upon, as time can be defined as a method of defining events relative to one another. This paper provides an insight into Time Dilation details of its occurrence, and the controversial representation of time.

GJSFR-A Classification: FOR Code: 02039p

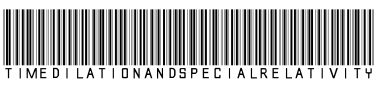

Strictly as per the compliance and regulations of:

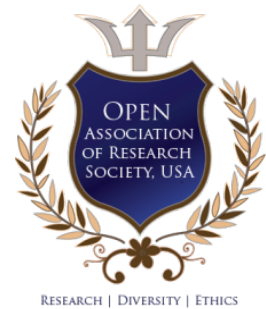

(C) 2021. Svanik Garg. This research/review article is distributed under the terms of the Attribution-NonCommercial-NoDerivatives 4.0 International (CC BY-NC-ND 4.0). You must give appropriate credit to authors and reference this article if parts of the article are reproduced in any manner. Applicable licensing terms are at https://creativecommons.org/licenses/by-nc-nd/4.0/. 


\section{Time Dilation and Special Relativity}

\section{Svanik Garg}

Abstract- The presentation and defination of Time as a concept in the Theory Special Theory of Relativity is remarkably difference to that of Time in the Theory of Classical Mechanics. In Special Relativity, Time is considered as the fourth dimension in the fabric of space-time. However, the presentation of time as such, can be argued upon, as time can be defined as a method of defining events relative to one another. This paper provides an insight into Time Dilation details of its occurrence, and the controversial representation of time.

\section{INTRODUCTION}

A Ibert Einstein mentioned in his Special Theory of Relativity that we exist in a 4 dimensional world, which has 3 space dimensions and a fourth dimension often called 'Spacetime'. The theory further explains that the motion of a reference frame (primary object) in the 'space' dimension reduces the passage through 'time' dimension which results in time progressing slower from the perspective of a reference frame, in motion.

However, this understanding of time and its dilation, Einstein presented, can be argued upon, given the structural flaws in the theory. Time can be comprehended to be a measure of duration of events, measured by the pace at which some activities happen". Eg- the vibration of an atom is an activity, and we measure time using this activity in an atomic clock.

\section{Key Concepts}

\section{a) Time}

'Time' can be described in simple terms to be a measure of motion with respect to frame of references. As said above, Time is just a measure of duration of events, measured by the pace at which some activities happen.

\section{b) Theory of Special Relativity}

Special relativity is an explanation of how speed affects mass, time and space, reflecting upon ideas such as time dilation, celeritas etc.

\section{c) Time Dilation}

A phenomenon, which exists due to the relativity between time and motion. It occurs, when under particular circumstances almost all activities slow down as the velocity of the specific frame of reference increase.

\footnotetext{
Author: The Doon School. e-mail: svanik.295.2024@doonschool.com
}

d) Frame of reference

An arbitrary position with reference to which the position or motion of something is described or physical laws are formulated.

\section{ili. Occurance Of Time Dilation}

The passage of time, and resultant dilation exists, specifically in the following cases

1. When the frame of reference is motile, or constantly changing position

2. When a force acts upon the frame of reference.

a) Case 1 - When the frame of reference is motile

Time is measured by the rate at which some activities happen, these activities which help quantify the concept of time are always based on motion. In the case of atomic clocks we measure time by vibrations of a particular atomic particle. Vibration is a repeated motion, and thus it can be proportionally reduced by the linear motion of the entire frame of reference. Therefore, the Time we observe in a motile reference frame runs slower than Time measured in a stationary reference frame. The reason behind such an occurence is that, Time Dilation as a concept takes place when there is a relative motion between the fabric of space and the reference frame, specifically when they converge or diverge. This is quite rare however, as the fabric of space provides opposition to the motion or activity of the reference frame, meaning it can only take place at speeds upwards that of light, as explained by Stephen Hawking. However, gathering evidence for such an occurence of time dilation is nearly impossible with our current physical understanding. It is known that light travels at the speed of $\mathrm{c}$, where $\mathrm{c}$ equals $299,792,458 \mathrm{~m} / \mathrm{s}$. The speed of light in vaccum is unknown to us, as any mass will be able to travel at any projectile velocity, however on the contrary it is impossible to cross the ' $c$ ' barrier for a given mass in our physical enviroment. That's because the fabric of space itself restricts light from travelling any faster than $c$, and the space-time fabric also restricts any massive body from travelling at the speed of light, making it physically impossible to cross the speed of light and test this specific occurence of Time Dilation.

This specific case of time dilation, contradicts Einstein's entire hypothesis pertaining to the movement of a projectile in reference to another which acts a frame of reference. If the projectile can move in light speeds, it means time is defined to be a dimension and not a measure of events, which theoretically should be incorrect. The equations of time dilation remain the 
same as that of Special theory of Relativity, as in both cases the equations are derived considering speed of light as the base value. It can be concluded that if, the space time fabric which supports the entire universe, itself prevents any matter from travelling at $\mathrm{c}$ or faster, the greatest speed possible should be said as 'Speed of light (c) with respect to the fabric of non-empty space(whose existence is debatable)'

Note: This case of Time Dilation by motion does not happen in absolute lack of matter/ vaccumm, however the existence of such a senario is impossible to judge, given the lack of knowledge we have pertaining to the theoretical existence of such a place without any matter or gravitational field.

b) Case 2 - When a linear force acts upon the frame of reference

This specific case can be defined using a simple analogy as outlined below: A particle is present on a spaceship, with a force acting on the spaceship and everything inside it. Then the clock inside spaceship ticks slower, in comparison to others. This happens due to the force, which is acting on the reference frame and all the matter in it, meaning the measure of time is altered completely. This case of Time Dilation is visibly seen if the force acting upon the object is strong enough to accelerate it to light speeds.

\section{i. Dilation in case of Inertial Forces}

Inertial force (sometimes called a pseudo force) is one such force that acts upon all. Like a gravitational force, when the inertial force acts on a particular object, it acts on every single component of matter in it inturn affecting, the time in that particular reference frame only. So, an inertial force acting upon this particular reference frame will always slow down the time (usually measured with a clock) inside one of the references frame. Centrifugal force is also another example of an inertial force, which acts in an identical manner and hence affects the time causing time dilation to take place

In order to test 'Time Dilation by Force (the second case)' as mentioned in this paper, it is possible to tie/attach any particular synchronized atomic clock to a wing of a rotating fan in the absence of any field such as a magnetic or gravitational field, as a centrifugal force will be acting upon all the matter present equally, creating a state where no external forces exist, suitable for Dilation to occur.

\section{ii. Specific Case}

\section{a. Opposite Forces}

A particular exception in the occurence of Time Dilation is when two different forces specifically act upon every specific particle inside the reference in absolute opposite direction, with the condition that, they cancel each other oout completely i.e resultant force has to be absolute zero. In such a situation, the factor by which Time slows down may not be always same, given the presence of two distinct forces as compared to the case when the resultant force is non-zero, and a single force acts upon the object as mentioned above. The time dilation factor in such a situation will also vary based on the resultant magnitude of these forces, however the principle of time dilation remains the same

\section{b. Freely fall}

When the frame of reference, through which time is measured, falls under the uniform acceleration of gravity, time dilation will neccesarily occur. However, in this case, Time Dilation will be very infinitesimal \& negligible (very hard to measure), given the limited force due to the gravitational field, and the subsequent limited accelaration. In case of a black hole, where the field strength is considreably stronger, theoretically time dilation will exist in quantifiable terms.

\section{Limitations}

1. It can be concluded that time dilation by motion, as mentioned in 3.1 will not happen in nothingness where there is NO force, and external factors at an absolute zero, which refers to the lack of mass or gravitational field or any other field or any other equivalent of mass, like antimatter. This is becuase, field has its own mass and a field like magnetic and gravity is a property of mass and not the emptiness, space itself. At this point of time, it is unknown whether such place exists in our known realm, however the existence of such space in our universe

2. In case 3.1 or time dilation, if the direction of unidirectional action is parallel to the direction of motion, the activity will rarely slow down.

3. In case of time dilation occuring due to a force, as described in 3.2, time slows down when the direction of force is contradcting the direction of the object itself. If the direction of force doesnt follow this property then the likelihood of time slowing down is very limited. This case of time dilation is unidirectional only ie ball will move in one direction ONLY, it is NOT a back and forth motion of the ball]. In the rare ocassion when there is a force on the medium itself (and on everything inside it, refer 3.2) in the direction of linear motion of the ball, then the ball would rarely ever slow down relative to an observer inside spaceship. However, any other activities inside the medium may slow down, given the same direction

\section{Experiment TO Test 'Time Dilation By Force'}

\section{a) Apparatus required}

- synchronized atomic clocks

1. Rotating fan, with even mass to which one of the clocks can be attached. 


\section{b) Methodology}

Attach an atomic clock to the blade of fan and rotate the fan for exactly 2.5 minutes, the fan should rotate with a very high RPM upwards of 800 (rotations per minute). During this time period, the needle (locus arm) of the rotating clock (attached to fan's wing) will be as close to a perfect circle as possible and the faster the velocity of ciruclar rotation more will be the

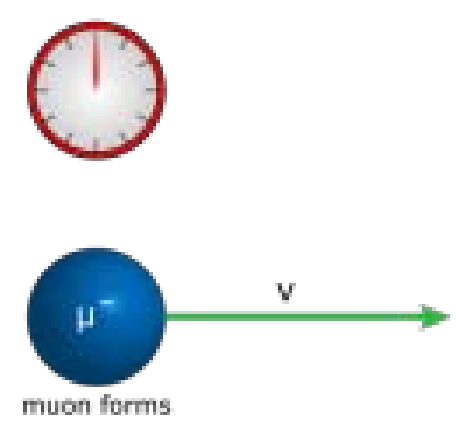

centrifugal force acting upon the relevant reference frame, the atomic clock in this specific case. The centrifugal force acting uniformly, will slow down the vibration inside of the atomic clock, which is attached to a fan. When this specific slowing down in vibration is accurately measured, the slowing down of clock and hence slowdown of time can be easily observed, reflecting upon the existence of time dilation.

Fig. 1: Alternative method to test the hypothesis Radioactive Decay

\section{c) Simulation}

The experiment was simulated using the generic procedure, where a digital clock accurate to 20 decmial places, a GPS and an altimeter is used to potray an atomic clock. The clock used was Van online simulation of an atomic clock which follows the same procedure: https://wwv.mcodes. org. Using a CFD simulation of a rotating fan an existing experiment on time dilation was used, and the slowing down of time was observed on the software: CFD

\section{d) Conclusion of the experiment}

The known effect, as measured many times in history suggest that a force acting upon the entire reference frame does cause Time Dilation, aforementioned in Case 2 of Time dilation

e) Observations

1. A shorter wing when simulated for the fan results in a higher centrifugal force providing an even more accurate and notable outcome. In this case the time dilation by case 1 is much less likely as compared to time dilation by centrifugal force.

2. The experiment has to be done as a simulation, as for accurate results the effect of gravity should be negligible, and therefore doing it artificially is the most accurate option.

3. Gravitational Time dilation, which was described in Case 1, is a result of case 2, which is due to a force, not due to einsteins very explanation suggesting the bending of space-time, and paradoxical alterations to the fabric.

4. The Third case of Time Dilation (through a regular medium), not really discussed in this paper also contributes to dilation in the above simulation, however its effect is next to nothing given the presence of hypersimulated conditions in this experiment. Furthermore, this specific case of Time Dilation by Medium also affects all the reference frames of this experiment making it irrelavant in this specific experiment. 


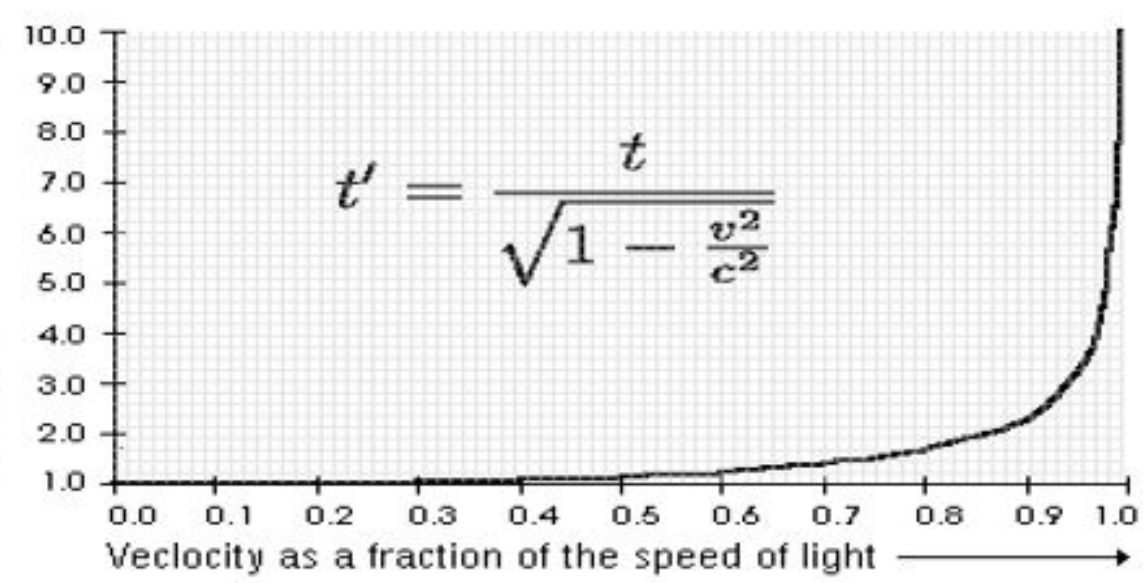

Fig. 3: Results obtained

\section{FINDINGS}

1. Time Dilation can occur as a result of 'Force'

2. Time is just a scalar measure of rate and longevity of events and can be simply measured by the rate at which some events take place. These actions can range from the vibrations of strings as part of the string theory, to the vibration of quarks and leptons to those of tangible atoms which consitute our matter. Therefore as defined by foreign entities is simply, 'slowing down of these activities under some circumstances relative to time'.

3. Due to the occurence of time dilation it can be understood, that time can be defined as an element of classical mechanics, contrary to that of einsteins' defination which stated that time is a seperate dimension.

\section{Vil. Contradictions}

1. Einstein's Special theory of Relativity 1 stated that, Time is a separate dimension. However, given this specific case of time dilation's existence it proves the hypothesis that, Time is just a measure of duration of events measured by the relative pace at which these actions take place, as if it existed in a seperate dimension its effect wouldnt be altered due to forces such as the centrifugal force in this case.

2. According to the special theory of relativity, Time Dilation is a result of movement in one of the space dimensions, relative to that the reference frame is present in or it is a result of the curvature or change in the space time fabric, however this experiment shows the contrary. It can be seen that time dilation is a result of a specific 'force' like in this experiment a centrifugal force acts upon on the reference frame and everything it contains.

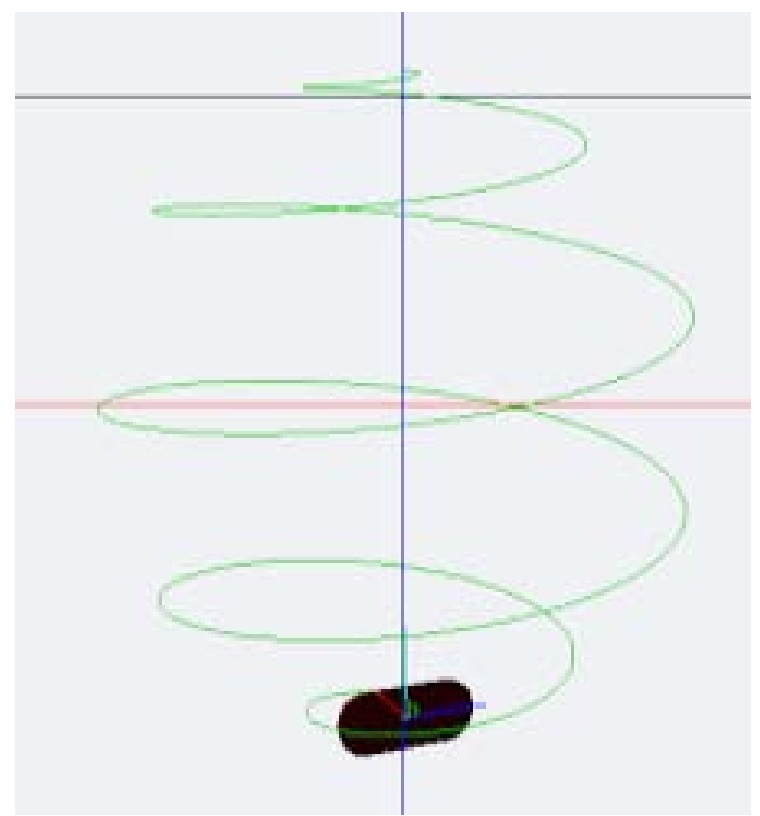

Fig. 4: Representation of time dilation in reference to eliptical movement

3. As per the third section of the Special theory of Relativity, Time Dilation can occur at all times where the reference frame is in motion, regardless of other external conditions except the enactment of a secondary force. However, as explained above, time dilation by motion, backed by classical mechanics, can be hypothesized to not happen in a state where matter ceases to exist and no field relays its effect. This is because the probability of the effect of time dilation on the specific particle or reference frame, is defined by matter, and therefore would become null and void otherwise.

4. Lastly, according to Special theory of Relativity1, Time shall always run in the polar opposite direction at a negative velocity much faster than that of light. 
However, Einstein also stated that it is impossible for any object to gain velocity that would exceed the limit of 'c' or rather be faster than the speed of light. However, it can be said that if speeds faster than light existed, time would not reverse itself, as time will move along at these speeds, given it is just a way of defining the rate at which events take place.

\section{References Références Referencias}

1. Einstein A., "Zur Elektrodynamik bewegter Körper" ("On the Electrodynamics of Moving Bodies"), Ann. Phys., 17, (1905).

2. James Clerk Maxwell. "A Dynamical Theory of the Electromagnetic Field," Phil. Trans. Royal Soc. 155 (1865).

3. http://www.ijsrp.org/research-paper-0914/ijsrpp3334.pdf

4. IOPsciencehttps://iopscience.iop.orgrarticle sabce 02 , pdf

5. https://www.academia.edu/Documents/in/Time Dilation

6. https://arxiv.org/abs/1707.00171

7. https://www.researchgate.net/publication/32056764 1_Is_Time_Dilation_a_scientific_theory

8. https://www.ijser.org/researchpaper/The ScientificConceptualization-of-Time-dilation.pdf

9. https://philpapers.org/rec/DIKDOG

10. https://www.sryahwapublications.com/openaccess-journal-of-physics/pdf/v4-i2/4.pdf

11. http://www.journalcra.com/article/length-contraction -time-dilation-gravity-total-existence-universe-andbeyond

12. https://arcticstardesign.com/2018/11/28/frames-ofreference/

13. https://www.science.org/doi/10.1126/sciadv.aax896 6

14. https://www.sciencedaily.com/releases/2020/10/201 023082010.htm

15. https://www.vedantu.com/physics/time dilation 